\title{
Gemtuzumab ozogamicin in acute myeloid leukemia: act 2, with perhaps more to come
}

\author{
Johann Hitzler ${ }^{1}$ and Elihu Estey ${ }^{2}$ \\ ${ }^{1}$ Division of Hematology/Oncology, The Hospital for Sick Children and Developmental and Stem Cell Biology, The Hospital for \\ Sick Children Research Institute, Toronto, ON, Canada and ${ }^{2}$ Division of Hematology University of Washington Medical Center \\ and Clinical Research Division Fred Hutchinson Cancer Research Center Seattle, WA, USA
}

E-mail:eestey@u.washington.edu doi:10.3324/haematol.2018.205948

T his editorial discusses two papers, both published in this issue of Haematologica, that expand our knowledge regarding gemtuzumab ozogamicin (GO), both in adults with newly-diagnosed acute myeloid leukemia (AML) and in children with relapsed AML. GO combines the toxin calicheamicin with an antibody to CD33, an antigen found on the surface of hematopoietic cells, including in some instances "stem cells" (Figure 1). ${ }^{1}$ The relative lack of CD33 expression on the surface of non-hematopoietic cells, except for macrophages lining hepatic sinusoids (Kupffer cells), limits the drug's principal toxicity to blood forming cells and to a lesser extent Kupffer cells; the latter toxicity results in a sinusoidal obstructive syndrome, most often seen after allogeneic hematopoietic cell transplant (HCT) but largely preventable. $^{2}$ On September 1, 2017, having originally approved but subsequently withdrawn approval for GO, the US Food and Drug Administration (FDA) re-approved use of GO combined with daunorubicin and cytosine arabinoside (ara-C) given as standard " $7+3$ " for treatment of adults with newly-diagnosed CD33-positive AML, who constitute the great majority of patients with the disease, thus officially ushering in GO's second act. (https://www.fda.gov/drugs/informationondrugs/approveddrugs/ucm574518.htm). Several months later, the European Medicines Agency (EMA) approved GO for the same indication (https://www.ema.europa.eu/medicines/human/ EPAR/mylotarg-O\#authorisation-details-section). The FDA also gave notice of its approval of GO for use as single agent in relapsed or refractory AML and in children.

Both the FDA and EMA approvals primarily rested on a French trial (ALFA-0701) randomizing newly diagnosed patients aged $50-70$ years to receive $7+3 \pm \mathrm{GO} 3 \mathrm{mg} / \mathrm{m}^{2}$ on days 1,4 , and $7 .^{3}$ In a break with precedent, which we discuss below, approval was based on prolongation of eventfree survival (EFS) rather than "overall" survival (hereafter "survival"); the benefit was limited to patients with "favorable" or "intermediate" cytogenetics. Median survival in patients remaining alive was 20 months. It is known, however, that the risk of relapse or death declines precipitously only once 2-3 years have elapsed since achievement of CR. ${ }^{4}$ Hence the update of the ALFA-0701 trial reported by Lambert et al. in the current issue of Haematologica is particularly noteworthy given the median follow up of 47 months in the $7+3+\mathrm{GO}$ group and 41 months in the controls. ${ }^{5}$ The essential findings of the original study remain unchanged, validating the FDA and EMA decisions to grant approval for GO.

Another report in the current issue of Haematologica by Niktoreh et al. from the Berlin-Frankfurt-Münster study group notes that when GO was given on a "compassion- ate" basis, either alone or with cytarabine, treatment resulted in sufficient reduction in blast count to permit $64 \%$ of 76 children aged under 18 years to receive HCT. At four years, probability of survival was $18 \pm 5 \%$ : $27 \pm 7 \%$ in the HCT and $0 \%$ in the non-HCT group. Although there is less experience with GO in children than in adults, this report follows a randomized trial of chemotherapy with or without addition of $\mathrm{GO}$ (one dose of $3 \mathrm{mg} / \mathrm{m}^{2}$ given on the first and fourth course of chemotherapy) conducted in over 500 children by the Children's Oncology Group (COG). ${ }^{7}$ Results were analogous to those reported in ALFA-0701: a greater effect on EFS than on survival and largely limited to individuals with intermediate or favorable cytogenetics. Both the COG study and the study reported here by Niktoreh et al. ${ }^{6}$ justify the FDA's approval of $\mathrm{GO}$ in children. The value of $\mathrm{GO}$ in relapsed pediatric AML will be clarified by the authors' ongoing prospective randomized controlled trial (EudraCT Number: 2010018980-41).

For many years, an increase in survival had been the sole basis for approval of new drugs in AML. This was sensible, since once failure to enter CR or relapse from CR had occurred, survival was typically limited to 2-3 months. Hence EFS and survival were largely synonymous. However today, the ability to keep people alive once these events have occurred has improved. Probably this is mainly due to improved supportive care, particularly better anti-fungal agents, but also reflects better "salvage" therapies. For example, the NCRI/MRC group in the UK reported that AML patients with intermediate-risk cytogenetics who did not receive HCT in first complete remission (CR1), nonetheless had similar survival as patients receiving HCT in CR1. ${ }^{8}$ This was a result of the ability to achieve, and then perform HCT in CR2 in the patients in whom relapse occurred after failure to perform HCT in CR1. The severance of EFS time from survival time has important implications. Once an event (no CR or relapse) occurs in a patient randomized to one arm of a phase III trial, there is no assurance that the salvage therapy or supportive care received will be identical to that received by a patient who has an event in the other arm of the trial. Hence what began as a randomized trial degenerates into an observational study, with all the attendant biases. Thus EFS, the primary end point in the ALFA-0701 trial, may be more reliable than survival as an indicator of the efficacy of an intervention such as GO. ${ }^{9}$

While there was an improvement in survival in the GO arm of ALFA-0701 and in the individual patient metaanalysis performed by Hills et al., ${ }^{10}$ that included ALFA0701, these improvements were not "statistically significant", unlike those in EFS, using $P<0.05$ as the criterion for 


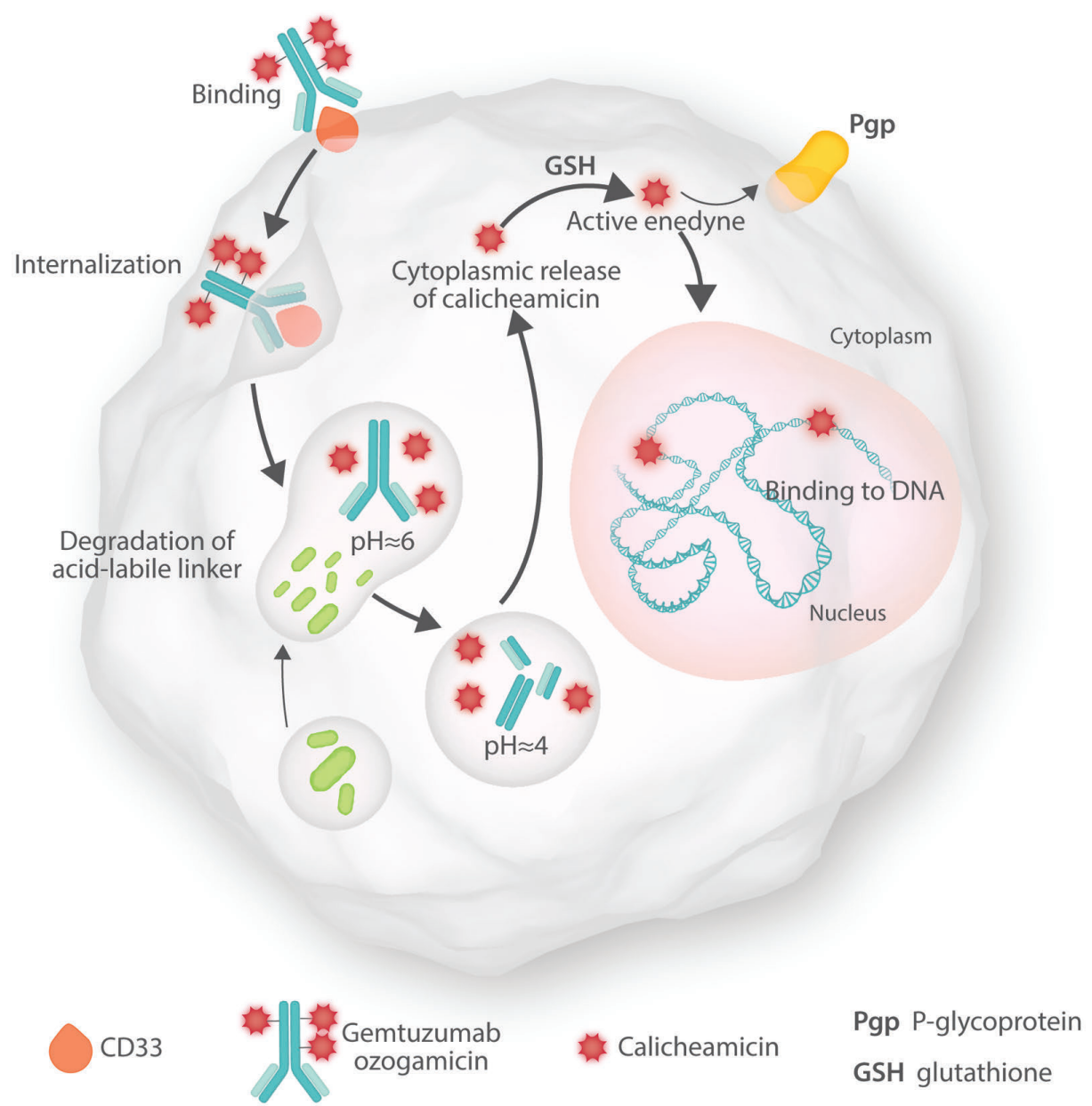

Figure 1. Mechanism of action of gemtuzumab ozogamicin.

statistical significance. However, comparison of hazard rates using $P$-values focuses on the relative value of an intervention rather than the absolute value. A good way to examine the latter is as the "number needed to treat" (NNT) to prevent a single event (or a single death); this information is often omitted from reports of clinical trials. Here, however, Lambert et al. note that at one year, 6 people would need to be treated with GO to prevent one event, while at year 3, NNT was 4. Such NNT values imply that most people do not benefit from GO when it is added to $7+3$.

Can we identify who will benefit? Since GO binds to CD33 it seems plausible that higher levels of CD33 expression might be associated with a better outcome following use of GO. Likewise, since it is known that GO, like agents such anthracyclines, is extruded from AML blasts by ATP-binding cassette transporter proteins mediating multidrug resistance (MDR), there may also be a relationship between extrusion and response to GO. Pollard et al. noted that children in the COG study who were in the highest $75 \%$ of CD33 expression had superior EFS regardless of cytogenetic group when given GO but not in the control group (no GO), ${ }^{11}$ and similar findings have been reported in adults for patients with favorable and intermediate cytogenetics. ${ }^{12,13}$ A higher dose (e.g. 6 $\mathrm{mg} / \mathrm{m}^{2}$ rather than $3 \mathrm{mg} / \mathrm{m}^{2}$ ) may benefit patients with low, but not high, CD33 expression. ${ }^{13}$ It should be kept in mind that quantification of neither CD33 expression nor $\mathrm{MDR}$ is currently a routine task. Attempts to enhance GO activity by use of cyclosporine A to block MDR do not appear to have been successful. ${ }^{14}$

More recently, attention has focused on the role of CD33 single nucleotide polymorphisms (SNPs) in affecting response to GO. Such SNPs regulate the expression of CD33 isoforms. SNPs denoted as TT result in a lack of exon 2 , resulting in absence of the antibody-binding site for $\mathrm{GO}$ on $\mathrm{CD} 33$, as well as in diagnostic immunophenotypic panels. Using data from the aforementioned COG study, Lamba et al. found only children with the CC SNP (51\% of 816 children), which encodes a full exon 2 resulting in the presence of a GO binding site on CD33, benefitted from addition of $\mathrm{GO}$ to chemotherapy regardless of cytogenetics or CD33 expression. ${ }^{15}$ However, this finding has not been confirmed by the UK NCRI/MRC group, ${ }^{16}$ although a similar proportion of patients (47\% of 563 patients) had the CC SNP as in Lamba et al., ${ }^{15}$ and the 
favorable cytogenetic group had the expected longer survival when given chemotherapy + GO rather than chemotherapy alone. The Southwest Oncology Group has also failed to notice an effect of CD33 SNPs on outcome in adults (M Othus, 2018, personal communication).

Even the minority of patients who benefit from GO might benefit more from development of improved antiCD33 therapeutics. ${ }^{17}$ One possibility here is use of bispecific antibodies (BiAbs) that engage CD33 but also direct $\mathrm{T}$ cells toward AML cells. An obvious model for this approach is blinatumomab, ${ }^{18}$ a CD3/CD19 molecule built in the Bispecific T-cell Engager (BiTE) format. A first series of CD33/CD3 BiAbs, including the BiTE AMG 330 and the tandem diabody AMV-564, have recently entered clinical tests.

Like GO, all CD33 BiAbs (and other CD33-directed therapeutics) currently under investigation recognize the $\mathrm{V}$ set domain, which is located distally on CD33. However, preliminary studies with artificial CD33 molecules show that membrane proximal binding enhances the immune effector cell functions of CD33 antibodies $(R$ Walter, 2018, personal communication). Development of antibodies recognizing such proximal sites is likely to be an area of examination in GO's second and, hopefully, subsequent acts.

\section{References}

1. Walter R, Appelbaum F, Estey E, and Bernstein I Acute myeloid leukemia stem cells and CD33-targeted immunotherapy. Blood. 2012;119(26):6198-6208.

2. McKoy J, Angelotta C, Bennett C, et al. Gemtuzumab ozogamicin-associated sinusoidal obstructive syndrome (SOS): an overview from the research on adverse drug events and reports (RADAR) project. Leuk Res. 2007;31(5):599-604

3. Castaigne S, Pautas C, Terré C, et al. Effect of gemtuzumab ozogamicin on survival of adult patients with de-novo acute myeloid leukaemia (ALFA-0701): a randomised, open-label, phase 3 study. Lancet. 2012;379(9825):1508-1516.

4. de Lima M, Strom S, Keating M, et al. Implications of potential cure in acute myelogenous leukemia: development of subsequent cancer and return to work. Blood. 1997;90(12):4719-4724.
5. Lambert J, Pautas C, Terré C et al. Gemtuzumab ozogamicin for de novo acute myeloid leukemia: final efficacy and safety updates from the open-label, phase III ALFA-0701 trial. Haematologica. 2019;104(1):113-119.

6. Niktoreh N, Lerius B, Zimmermann M et al. Gemtuzumab ozogamicin in children with relapsed or refractory acute myeloid leukemia: a report by Berlin-Frankfurt-Münster study group. Haematologica. 2019;104(1):120-127.

7. Gamis AS, Alonzo TA, Meshinchi S, et al. Gemtuzumab ozogamicin in children and adolescents with de novo acute myeloid leukemia improves event-free survival by reducing relapse risk: results from the randomized phase III Children's Oncology Group trial AAML0531.J Clin Oncol. 2014;32(27):3021-3032.

8. Burnett AK, Goldstone A, Hills RK, et al. Curability of patients with acute myeloid leukemia who did not undergo transplantation in first remission. J Clin Oncol. 2013;31(10):1293-1301.

9. Estey E, Othus M, Lee SJ, Appelbaum FR, Gale RP. New drug approvals in acute myeloid leukemia: what's the best endpoint? Leukemia. 2016;30(3):521-525

10. Hills RK, Castaigne S, Appelbaum FR, et al. Addition of gemtuzumab ozogamicin to induction chemotherapy in adult patients with acute myeloid leukaemia: a meta-analysis of individual patient data from randomised controlled trials. Lancet Oncol. 2014;15(9)986-996.

11. Pollard JA, Loken M, Gerbing RB, et al. CD33 Expression and Its Association With Gemtuzumab Ozogamicin Response: Results From the Randomized Phase III Children's Oncology Group Trial AAML0531. J Clin Oncol. 2016;34(7):747-755.

12. Olombel G, Guerin E, Guy J, et al. The level of blast CD33 expression positively impacts the effect of gemtuzumab ozogamicin in patients with acute myeloid leukemia Blood. 2016;127(17) 2157-2160.

13. Khan N, Hills RK, Virgo P, et al. Expression of CD33 is a predictive factor for effect of gemtuzumab ozogamicin at different doses in adult acute myeloid leukaemia. Leukemia. 2017;31(5):1059-1068.

14. Tsimberidou A, Estey E, Cortes J, et al. Gemtuzumab, fludarabine, cytarabine, and cyclosporine in patients with newly diagnosed acute myelogenous leukemia or high-risk myelodysplastic syndromes. Cancer. 2003;97(6):1481-1487.

15. Lamba JK, Chauhan L, Shin M, et al. CD33 Splicing Polymorphism Determines Gemtuzumab Ozogamicin Response in De Novo Acute Myeloid Leukemia: Report From Randomized Phase III Children's Oncology Group Trial AAML0531. J Clin Oncol. 2017;35(23):26742682.

16. Gale R, Pope T, Wright M, et al. No evidence that CD33 splicing SNP impacts the response to GO in younger adults with AML treated on UK MRC/NCRI trials. Blood. 2018;131(4):468-471.

17. Walter $R$ Investigational CD33-targeted therapeutics for acute myeloid leukemia. Expert Opin Investig Drugs 2018; 27(4): 339-348

18. Kantariian H, Stein A, Gökbuget N, et al. Blinatumomab versus Chemotherapy for Advanced Acute Lymphoblastic Leukemia. N Engl J Med. 2017;376(9):836-847.

\section{Lenalidomide can be safely combined with chlorambucil and rituximab in older patients with chronic lymphocytic leukemia}

\section{Candida Vitale ${ }^{1}$ and Alessandra Ferrajoli ${ }^{2}$}

'Division of Hematology, University of Torino, A.O.U. Città della Salute e della Scienza di Torino, Italy and 'Department of Leukemia, The University of Texas MD Anderson Cancer Center, Houston, TX, USA

\section{E-mail:aferrajo@mdanderson.org}

doi:10.3324/haematol.2018.206359

T the clinical activity of lenalidomide in chronic lymphocytic leukemia (CLL) was first reported more than 10 years ago. ${ }^{1,2}$ Since then, this agent has been studied in various combinations with anti-CD20 monoclonal antibodies, chemotherapy, chemo-immunotherapy and B-cell receptor (BCR)-targeting agents. These studies have shown clinical responses; however, most importantly, they have also highlighted unique and unexpected toxicities, in particular when lenalidomide was combined with chemo-immunotherapy and targeted agents.

In this issue of Haematologica, Kater and colleagues report the experience of the HOVON CLL study group on the feasibility and efficacy of the combination of lenalidomide, chlorambucil, and rituximab in treatment-naïve patients 